\title{
Comparative molecular docking studies of EGCG with $\mathrm{SHaPrPC}$
}

\begin{abstract}
Epigallocatechin gallate (EGCG) is a naturally occurring alkaloid found in green tea. It has been shown to bind with nanomolar affinity to the monomeric prion protein and induce conformational instability. A better understanding of the specific molecular interactions that impart this strong interaction and molecular rearrangement are of interest for the development of ligands that possess both high affinity yet impart stabilizing effects. Recently, it has been demonstrated that molecules with such characteristics reduce $\mathrm{PrP}^{\mathrm{sc}}$ titers in both in-vitro and ex-vivo experiments. To gain structural insights into EGCG's effect on the monomeric prion protein $\left(\mathrm{PrP}^{\mathrm{c}}\right)$, comparative molecular docking studies of EGCG was performed against $\mathrm{SHaPrP}^{\mathrm{C}}$ using MOE, Sybyl and FlexX software docking programs. These results show that the side orientations of Tyr ${ }^{169}$ and $\mathrm{Tyr}^{218}$ play an important role in binding of EGCG near the amylome region motif between loop-2 and helix-2.
\end{abstract}

Keywords: docking, ECGC, $\mathrm{SHaPrP}^{\mathrm{C}}$
Volume 2 Issue 5 - 2018

\author{
Nataraj Sekhar Pagadala,' Rolando Perez- \\ Pineiro, ${ }^{2}$ Trent C Bjorndahl, ${ }^{2}$ David S \\ Wishart ${ }^{2}$ \\ 'Department of Medical Microbiology and Immunology, \\ University of Alberta, Canada \\ ${ }^{2}$ Departments of Biological Sciences, and Computing Science, \\ University of Alberta, Canada
}

Correspondence: Nataraj Sekhar Pagadala, Department of Medical Microbiology and Immunology, University of Alberta, Canada, Email nattu25।@gmail.com

Received: July 29, 2018 | Published: September 24, 2018

\section{Introduction}

The infectious prions can act as self-replicating agents. ${ }^{1}$ The disease progresses by conformational transition, utilizing cellular PrP $\left(\mathrm{PrP}^{\mathrm{C}}\right)$ as substrate. ${ }^{2}$ This transforms endogenous $\alpha$-helix-rich $\operatorname{PrP}^{\mathrm{C}}$ to $\beta$-sheet-rich, disease-causing proteolytic resistant conformations $\left(\mathrm{PrP}^{\mathrm{Sc}}\right) .^{3-7}$ However, recently it has been shown that $\mathrm{PrP}^{\mathrm{Sc}}$ forms amyloid fibrils some of which are protease sensitive. ${ }^{8-13}$ In vitro experiment on recombinantly expressed and refolded $\operatorname{PrP}(\mathrm{rPrP})$ show that EGCG (epigallocatechin-3-gallate) promotes the formation of random coil structure destabilizing the natively folded $r P r P$. These random coil conformers formed by EGCG were initially monomeric and further converted to PK-sensitive aggregates. ${ }^{14}$ It was also shown that EGCG elicits switching the susceptible form Sup35 prions to resistant forms In vivo. ${ }^{15}$ In this study, we show that epigallocatechin gallate (EGCG), the major polyphenol in green tea, bind to pocket $\mathrm{A}$ near amylome region of $\mathrm{SHaPrP}^{\mathrm{C}}$ and might induce rapid transition of $\mathrm{PrP}^{\mathrm{C}}$ into a detergent-insoluble conformation

\section{Methodology}

MOE 2009.10 (Chemical computing group, Montreal, Canada) and SybylX 1.3 (SYBYL-X 1.3, Tripos International, 1699 South Hanley Rd., St. Louis, Missouri, 63144, USA) were used for in-silico docking studies. Initially, in order to identify a suitable PrP model for docking, structures from NMR ensemble of Syrian hamster PrP further referred as SHaPrP (PDB ID: 1B10) were superimposed and an average structure was calculated by MOE. Pair-wise alignment of each model from the NMR ensemble with the average structure revealed that model $\# 17$ had the smallest RMSD of $0.5 \AA$. This model was energy minimized with CHARMM27 force field using generalized born implicit solvent representation. ${ }^{16}$ To take into account a possible role of PrP-bound water molecules in PrP-EGCG binding, water coordinates from $\mathrm{X}$-ray structure of human prion protein (PDB ID: 3HAF) were transferred to the model of hamster PrP. In order to achieve this, the hamster PrP model was superimposed with the X-ray structure of human PrP and coordinates of 26 water molecules from the X-ray structure were appended to coordinates of the superimposed hamster PrP model. MOE alpha site finder was used to identify $\mathrm{SHaPrP}$ binding pocket. The rotamer orientations of the residues in the binding pocket with a distance radius of $1.2 \AA$ that provided the maximal hydrophobicity of the binding pocket near the amylome region were predicted using rotamer explorer of MOE and were used further in docking simulations using MOE. For In silico docking in MOE, the ligand placement method "Alpha PMI" was employed to bias the conformational search of the ligand to meaningful trials by aligning and matching all triangles of the template points with compatible geometry using London $\mathrm{dG}$ scoring function. Monte Carlo method of simulated annealing was used to find the global minimum by exploring various states of a configuration space with small changes in the current state. Each new state is accepted or rejected according to Metropolis criterion. Binding poses, which were initially identified during the simulated annealing step, were further optimized by energy minimization with the Tripos Assisted Force Field for small molecules. Finally, relative binding free energies, electrostatic energy, vander Waals energy, and solvation energy were calculated (i.e., solvent electrostatic correction). Solvation energies were calculated using Poisson-Boltzmann equation. The reaction model dielectric function with a cut-off between 8 and $10 \AA$ and the pocket radius of $6.0 \AA$ was used for our docking studies. For In silico docking using Surflex X, an idealized active site ligand (called a protomol) is generated from the protein structure. The protomol construction is based solely on the hydrogen atoms of the protein residues that constitute the active site. The parameters used to produce small and buried target are proto_thresh $=0.5$ and proto_bloat $=0$. Ligand is docked in mol2 format using the whole molecule approach. The input ligand is fragmented into 1-10 molecular fragments with some rotatable bonds for each fragment. Each fragment is conformationally searched and a maximum of 100 conformations per fragment is generated in each stage of the incremental construction process. Each conformation of each fragment is aligned to the protomol to yield 
poses that maximize molecular similarity to the protomol. Finally, the aligned fragments are scored and pruned on the basis of the scoring function and the degree of protein interpenetration. The whole protein is considered in all the two docking programs. The binding poses with high affinity that were obtained from two different docking calculations were further minimized in the binding pocket with ligX program of MOE using TAFF force field. In order to compare results from different docking program in a uniform manner, stability of PrP-EGCG complexes was tested with molecular dynamics using the CHARMM27 force field distributed in MOE, starting from the fully minimized structures in the (NVT) ensemble at a temperature of $300 \mathrm{~K}$. An integration time step of $0.5 \mathrm{fs}$ was used. No restraints were applied to atomic positions. No explicit water was added and born solvation dielectric was used. The total simulation time was $10000 \mathrm{ps}$. The root-mean-square deviations (rmsd) of the ligand conformations in the binding pocket were calculated from each trajectory to quantify the stability of the protein-ligand complex.

\section{Results and discussion}

Out of thirty that were used as cut-off for blind docking study using MOE, only seventeen poses were able to bind with the negative binding free energy. Out of seventeen poses, only single pose prefers to bind near amylome region, four poses bind near pocket the socalled thiamine binding site and the remaining twelve poses bind in the binding pocketD near $\mathrm{Glu}^{196}$ where known inhibitor GN8 binds in MoSHaPrP. ${ }^{17-19}$ These results reveal that the largest cluster of EGCG prefer to bind GN8 pocket with the top pose binding with highest affinity of $-9.3 \mathrm{Kcal} / \mathrm{Mol}$ Figure $1 \mathrm{~A}$. Further, docking studies of EGCG was performed with the energy minimized structure of model \#17 in the presence of water and ions. The side chain orientations of $\mathrm{Tyr}^{169}, \mathrm{Phe}^{175}$ and $\mathrm{Tyr}^{218}$ containing aromatic rings that provided the maximal hydrophobicity of the binding pocket were also used in docking simulations. The RMSD of $\mathrm{C} \alpha$ between the native and energy minimized structures were $1.5 \AA$. Out of seventeen poses that bind with negative binding affinity, five poses bind to pocket A nearer amylome motif (NNQNNF) on the outer surface of $\mathrm{SHaPrP}^{\mathrm{C}}$, seven poses bind to pocket $\mathrm{B}$ between loop1 and $\alpha 1$ near $\mathrm{Tyr}^{150}$ that was identified as thiamine binding site, three poses bind to pocket $\mathrm{D}$ near $\mathrm{Glu}^{196}$ and two poses bind to pocket $\mathrm{C}$ near His ${ }^{186}$ Figure 1B. The top pose that prefers pocket A amylome region binds with high affinity of $-10.2 \mathrm{Kcal} / \mathrm{mol}$. These docking results on native and energy minimized structure of $\mathrm{SHaPrP}^{\mathrm{C}}$ (model\#17) reveals that EGCG binding shifts from pocket $\mathrm{D}$ (GN8 pocket) to pocket $\mathrm{B}$ (thiamine binding site) and pocket A (amylome motif region). Binding pocket analysis for the energy minimized structure showed pocket A (amylome motif region) was top ranked (Pocket size of $90 \AA$ and number of side chain atoms of 68 including fifteen hydrophobic atoms with higher number of solvent exposed atoms) Figure 1B. In the energy minimized model, the native orientation of $\mathrm{Tyr}^{169}$ side chain orients with $\chi^{1}$ of $75.1^{0}$ and $\chi^{2}$ of $76.6^{0}$ and Phe ${ }^{175}$ side chain orients with $\chi^{1}$ of $-172.3^{0}$ and $\chi^{2}$ of $-88.5^{0}$. Side chain analysis of $\operatorname{Tyr}^{169}$ showed three rotamers with $\chi^{1}$ and $\chi^{2}$ of 75 and $62^{\circ}, 93$ and $78^{\circ}$, and -83 and $-68^{\circ}$ respectively. The first two rotamers doesn't show any significant increase in the number of hydrophobic atoms of the binding pocket and are not considered further. But rotamer 3 allows the side chain to orient in the opposite direction compared to native orientation and opens the new binding pocket with tight atomic packing between loop3 and helix 3 near the pocket A (amylome region) with the residues $\mathrm{Val}^{166}, \mathrm{Tyr}^{169}, \mathrm{Asn}^{170}$,
$\mathrm{Asn}^{172}, \mathrm{Gln}^{172}, \mathrm{Phe}^{175}, \mathrm{Gln}^{217}, \mathrm{Tyr}^{218}, \mathrm{Glu}^{221}$ and $\mathrm{Ser}^{222}$ (pocket size of 64 and side chain atoms of 41 including 12 hydrophobic atoms with less number of solvent exposed residues). Blind docking of EGCG performed against $\mathrm{SHaPrP}^{\mathrm{C}}$ with new side chain orientation for $\operatorname{Tyr}^{169}\left(\chi^{1}\right.$ of $-83^{\circ}$ and $\chi^{2}$ of $\left.-68^{\circ}\right)$ along with structural water and ions show all the thirty conformations occupy the new binding pocket $\mathrm{A}$ between loop3 and helix 3 near the amylome region Figure $2 \mathrm{~A}$. The high affinity conformation with $-14.6 \mathrm{Kcal} / \mathrm{mol}$ show one hydrogen bonding and two hydrophobic interactions with $\operatorname{Ser}^{222}(\mathrm{~N}), \operatorname{Val}^{166}\left(\mathrm{C} \gamma^{2}\right)$ and $\mathrm{Phe}^{175}\left(\mathrm{C}^{2}\right)$ with $\mathrm{O} 6, \mathrm{C} 7$ and $\mathrm{C} 15$ atoms of the ligand respectively. The higher binding energy implies an important role that $\mathrm{Tyr}^{169}$ of $\mathrm{SHaPrP}^{\mathrm{C}}$ plays in binding EGCG. Rotamer optimization of $\mathrm{Phe}^{175}$ did not result in improved hydrophobicity of the binding pocket. Therefore, the native orientation of $\mathrm{Phe}^{175}$ side-chain was used in our docking studies. Overall, four side chain rotamers were predicted for $\mathrm{Tyr}^{218}$. First two rotamers corresponding to experimental structure do not show any significant increase in the number of hydrophobic atoms and size of the pocket and were not considered further. The third rotamer of Tyr ${ }^{218}$ with specific angles of $\chi^{1}$ of $-158^{\circ}$ and $\chi^{2}$ of $70^{\circ}$ (pocket size with 69 and side chain atoms with 43 including 12 hydrophobic atoms) show variations in the size and hydrophobicity of the binding pocket was used for further docking studies. Blind docking of EGCG in the presence of water and ions with new side chain orientations of $\mathrm{Tyr}^{218}$ allows to bind with a high affinity of $-15.9 \mathrm{Kcal} / \mathrm{mol}$ and $-14.7 \mathrm{Kcal} / \mathrm{mol}$ compared to optimized structure of $\mathrm{Tyr}^{169}$ Figure 2B. The conformation with high affinity when further minimized in the binding pocket using lig $\mathrm{X}$ with new side chain orientations of $\mathrm{Tyr}^{169}$ (rotamer3) and $\mathrm{Tyr}^{218}$ (rotamer3) show six hydrogen bonding and two hydrophobic interactions with $\mathrm{Asp}^{167}, \mathrm{Gln}^{172}, \mathrm{Gln}^{217}, \mathrm{Tyr}^{218}$, $\mathrm{Ser}^{222}, \mathrm{Tyr}^{225}$, $\mathrm{Val}^{166}$ and $\mathrm{Phe}^{175}$ residues respectively Table1. In order to ensure that MOE results are not an artifact of MOE scoring function, docking was also conducted using other commonly used program, such as Surflex with the final structure of $\mathrm{SHaPrP}^{\mathrm{C}}$ with new side chain orientations of $\operatorname{Tyr}^{169}$ (rotamer3) and $\mathrm{Tyr}^{218}$ (rotamer3). Docking studies carried out using Surflex also showed EGCG binding to the new binding pocket A between loop3 and helix 3 near the amylome region. Analysis of the top Surflex binding pose revealed that EGCG orientation in the binding pocket is stabilized by seven hydrogen bonds and two hydrophobic interactions between EGCG and $\mathrm{PrP}^{\mathrm{C}}$ residues $\mathrm{Pro}^{165}, \mathrm{Asn}^{171}, \mathrm{Gln}^{172}, \mathrm{Gln}^{217}, \mathrm{Tyr}^{218}, \mathrm{Ser}^{218}, \mathrm{Ser}^{222}, \mathrm{Tyr}^{225}$, $\mathrm{Val}^{166}$ and Phe ${ }^{175}$ Table 1 and Figure 3. These docking results based on MOE and surflex $\mathrm{X}$ reveals that the conformation generated by surflex $\mathrm{X}$ show more number of interactions with the amino acids involved in Pocket A. Binding free energy in terms of $\Delta \mathrm{G}$ calculated using Hyde module of Flex X to recalculate detla $\mathrm{G}$ for top binding poses from two different programs also showed that the conformation of EGCG generated by surflex X binds with high $\mathrm{G}$ of $-16 \mathrm{Kj} / \mathrm{mol}$. These results show that the conformation generated by surflex $X$ is the most probable conformation and the side orientations of $\mathrm{Tyr}^{169}$ and $\mathrm{Tyr}^{218}$ play an important role in binding of EGCG to pocket A near the amylome region motif between loop-2 and helix-2. Stability of EGCG-SHaPrP ${ }^{\mathrm{C}}$ complexes under a more accurate Generalized Born representation of solvation effects was studied with MOE molecular dynamics. Stability of EGCG-SHaPrPC binding poses was evaluated by calculating RMSD of the ligand as a function of simulation time. Molecular dynamics revealed that the top binding pose of EGCG$\mathrm{SHaPrP}{ }^{\mathrm{C}}$ complex generated by Surflex Figure $4 \mathrm{~A}$ is more stable than the top binding poses produced by MOE Figure 4B. 
Table I Ligand-protein contacts of ECGC top binding poses generated using MOE and SYBYLX 2.0 software suite near pocketA of SHaPrPC

\begin{tabular}{|c|c|c|c|c|}
\hline \multirow{2}{*}{ Software } & \multicolumn{2}{|c|}{ Ligand contacts before energy minimization } & \multicolumn{2}{|c|}{ Ligand contacts after energy minimization } \\
\hline & Hydrogen bonding & Hydrophobic & Hydrogen bonding & Hydrophobic \\
\hline \multirow{6}{*}{ MOE } & Arg164.O-O7Lig & Val166.Cg1-C14 & Asp167.Od1—O4Lig & Val166.Cg1—C5Lig \\
\hline & Pro165.O_O4Lig & Phe175.Ce2-C21 & Gln172.N—O6Lig & Phe175.Ce2-C3Lig \\
\hline & Val166.O-O4Lig & & $\mathrm{G} \ln 217 . \mathrm{O}-\mathrm{O} 3 \mathrm{Lig}$ & \\
\hline & Tyr169.O-O6Lig & & Tyr218.O-O3Lig & \\
\hline & Asn170.O-O6Lig & & Ser222.N_—O3Lig & \\
\hline & & & Tyr225.O_ O7Lig & \\
\hline \multirow{7}{*}{ Sybyl } & Asn171.Od1-OLig & $\begin{array}{l}\text { VAL166.CG1- } \\
\text { CLig }\end{array}$ & PRO165.O_OLig & VAL166.CG1_CLig \\
\hline & GLN172.N_—OLig & PHE175.CE2-CLig & ASN171.OD1-OLig & PHE175.CE2_-CLig \\
\hline & TYR218.O__ OLig & & GLN172.N_—OLig & \\
\hline & SER222.N_—OLig & & GLN217.O_ OLig & \\
\hline & & & TYR218.O__ OLig & \\
\hline & & & SER222.N_—OLig & \\
\hline & & & TYR225.O-OLig & \\
\hline
\end{tabular}
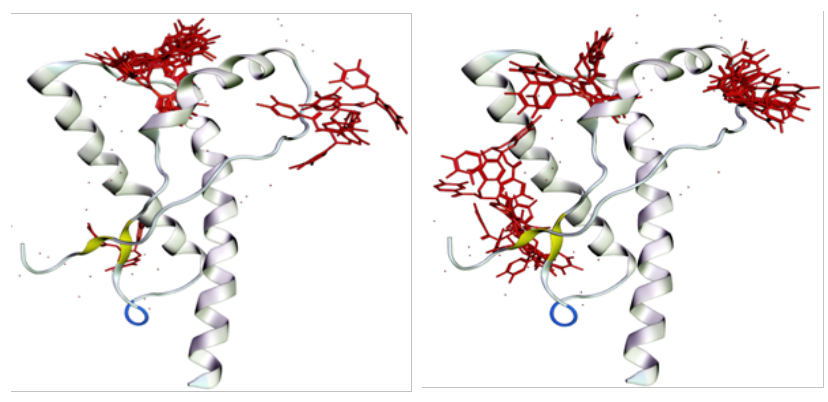

Figure I (A) Blind docking results of EGCG with SHaPLrPC NMR model (IBI0:I7), (B) for the energy-minimized structure of SHaPrPC (IBIO:I7) in the presence of water and lons.
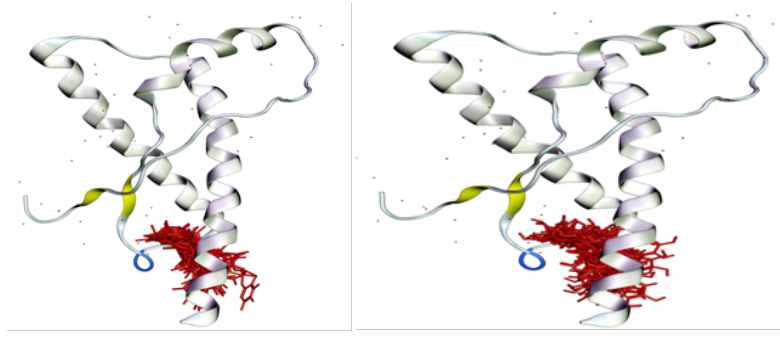

Figure 2 Blind docking of EGCG performed against $\mathrm{SHaPrP}^{\mathrm{C}}$ with new side chain orientation for $\operatorname{Tyr}^{169}\left(\chi 1\right.$ of $-83^{\circ}$ and 2 of $\left.-68^{\circ}\right)(\mathbf{A}) \operatorname{Tyr}^{218}(\mathbf{B})$ along with structural water and ions.

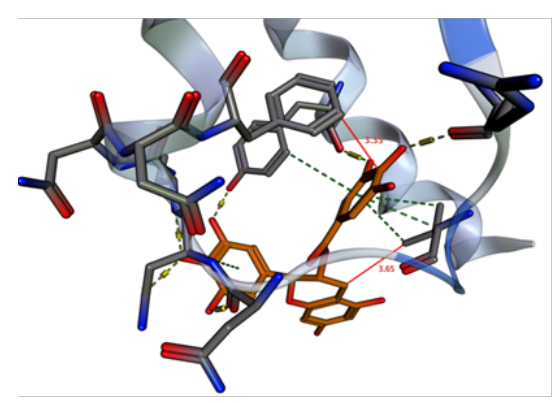

Figure 3 Ligand protein interaction of ECGC with $\mathrm{SHaPrP}^{\mathrm{C}}$ using surflex $\mathrm{X}$ docking program implemented in sybyl X 2.0 software suite.

$$
\text { EGCG_conformation_Sy by: }
$$

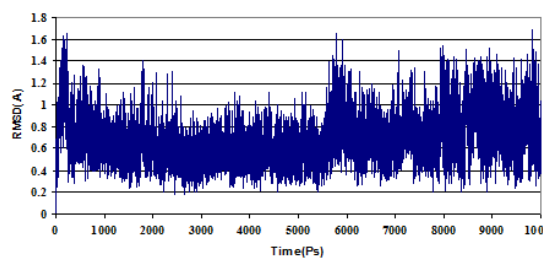

EGCG_conformation_MOE

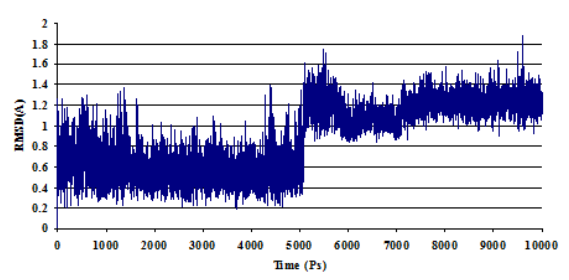

Figure 4 Calculated RMSD graphs of MD simulations for the top binding poses of ECGC-SHaPrPC generated using SybylX2.0 (A) and MOE (B) using NAMD software. Time (ps) is taken on $\mathrm{X}$-axis and RMSD on Y-axis. 


\section{Conclusion}

Molecular docking studies show that ECGC binds to pocket A between loop3 and helix3 of $\mathrm{SHaPrP}^{\mathrm{C}}$ interacting with $\mathrm{Tyr}^{169}$ and $\mathrm{Tyr}^{218}$.

\section{Acknowledgements}

I thank Dr.Wishart, Department of Computing Science and Biological Sciences, university of alberta, Edmonton, Canada, for providing the laboratory facilities and helpful discussions.

\section{Conflict of interest}

Authors declare that there is no conflict of interest.

\section{References}

1. Prusiner SB. Novel proteinaceous infectious particles cause scrapie. Science. 1982;216(4542):136-144.

2. Prusiner SB. Shattuck lecture-neurodegenerative diseases and prions. The New England journal of medicine. 2001;344(20):1516-1526.

3. Pan YT, Hori H, Saul R, et al. Castanospermine inhibits the processing of the oligosaccharide portion of the influenza viral hemagglutinin. Biochemistry. 1983;22(16):3975-3984.

4. Basler K, Oesch B, Scott M, et al. Scrapie and cellular PrP isoforms are encoded by the same chromosomal gene. Cell. 1986;46(3):417-428.

5. Bolton DC, Kinley MP, Prusiner SB. Identification of a protein that purifies with the scrapie prion. Science. 1982;218(4579):1309-1311.

6. Prusiner SB, Kinley MP, Bowman KA, et al. Scrapie prions aggregate to form amyloid-like birefringent rods. Cell. 1983;35(1):349-358.

7. Meyer RK, Kinley MP, Bowman KA, et al. Separation and properties of cellular and scrapie prion proteins. Proceedings of the National Academy of Sciences of the United States of America. 1986;83(8):2310-2314.

8. Safar J, Wille H, Itri V, et al. Eight prion strains have $\operatorname{PrP}(\mathrm{Sc})$ molecules with different conformations. Nature medicine. 1998;4:1157-1165.
9. Tzaban S, Friedlander G, Schonberger O, et al. Protease-sensitive scrapie prion protein in aggregates of heterogeneous sizes. Biochemistry. 2002;41(42):12868-12875.

10. Tremblay P, Ball HL, Kaneko K, et al. PrPSc conformers induced by a synthetic peptide and several prion strains. Journal of virology. 2004;78(4):2088-2099.

11. Legname G, Nguyen HO, Peretz D, et al. Continuum of prion protein structures enciphers a multitude of prion isolate-specified phenotypes. Proceedings of the National Academy of Sciences of the United States of America. 2006;103(50):19105-19110.

12. Pastrana MA, Sajnani G, Onisko B, et al. Isolation and characterization of a proteinase $\mathrm{K}$-sensitive PrPSc fraction. Biochemistry. 2006;45(51):15710-15717.

13. Colby DW, Zhang Q, Wang S, et al. Prion detection by an amyloid seeding assay. Proceedings of the National Academy of Sciences. 2007;104(52):20914-20919.

14. Rambold AS, Muller V, Ron U, et al. Stress-protective signaling of prion protein is corrupted by scrapie prions. The EMBO journal. 2008;27(14):1974-1984

15. Roberts BE, Duennwald ML, Wang H, et al. A synergistic smallmolecule combination directly eradicates diverse prion strain structures. Nature chemical biology. 2009;5(12):936-946.

16. Mackerell AD. Empirical force fields for biological macromolecules: overview and issues. Journal of computational chemistry. 2004;25(13):1584-1604.

17. Perez-Pineiro R, Bjorndahl TC, Berjanskii MV, et al. The prion protein binds thiamine. The FEBS journal. 2011;278(21):4002-4014.

18. Kuwata K, Nishida N, Matsumoto T, et al. Hot spots in prion protein for pathogenic conversion. Proceedings of the National Academy of Sciences of the United States of America. 2007;104(29):11921-11926.

19. Pagadala NS, Bjorndahl TC, Blinov N, et al. Molecular docking of thiamine reveals similarity in binding properties between the prion protein and other thiamine-binding proteins. Journal of molecular modeling. 2013;19(12):5225-5235. 